\title{
ELECTRONIC WORD OF MOUTH (E-WOM) E ESCOLHA DO DESTINO TURÍSTICO POR PESSOA COM DEFICIÊNCIA OU MOBILIDADE REDUZIDA (PCDMR)
}

\author{
Marklea da Cunha Ferst \\ Doutora em Turismo e Hotelaria pela Universidade do Vale do Itajaí \\ Universidade do Estado do Amazonas - UEA. \\ Manaus - AM / Brasil. \\ mferst@uea.edu.br \\ Sara Joana Gadotti dos Anjos \\ Doutora em Engenharia de Produção/Gestão de Negócios \\ pela Universidade Federal de Santa Catarina \\ Universidade do Vale do Itajaí. \\ Itajaí - SC / Brasil. \\ anjos.sara@hotmail.com \\ Helen Rita Menezes Coutinho \\ Mestre em Administração pela Universidade Federal de Santa Catarina. \\ Universidade do Estado do Amazonas - UEA. \\ Manaus - AM / Brasil. \\ hcoutinho@uea.edu.br \\ Luiz Carlos da Silva Flores \\ Doutorado em Engenharia de Produção pela Universidade Federal de Santa Catarina. \\ Universidade do Vale do Itajaí - Univali. \\ Itajaí - SC / Brasil. \\ luiz.flores@univali.br
}

\section{Resumo}

Objetivo do estudo: Analisar o impacto do $e-W O M$ na escolha do destino turístico por pessoa com deficiência ou mobilidade reduzida (PcDMr).

Metodologia/abordagem: Trata-se de um estudo de natureza explicativa; dedutivo e quantitativo com a utilização de uma amostra não probabilística por conveniência, com 128 pessoas com deficiência ou mobilidade reduzida. A análise de dados foi realizada com auxílio do software SPSS Statistics versão 25. Utilizou-se métodos de estatística descritiva para a análise dos dados. Análise do Teste-T e correlação entre variáveis.

Originalidade/Relevância: Os estudos acadêmicos sobre o uso do $e$-WOM existentes não abordam a sua utilização por pessoas com deficiência ou mobilidade reduzida. A relevância acadêmica se dá face o crescimento do mercado do turismo acessível e da necessidade de conhecer melhor os hábitos deste segmento.

Principais resultados: Observou-se que a maioria dos respondentes com deficiência ou mobilidade reduzida viajam acompanhados e ao menos uma vez ao ano. Quanto a acessibilidade do destino, para os turistas PcDMr esta tem influência ou pode influenciar na escolha do destino turístico. No tocante ao uso das redes sociais a maioria dos respondentes PcDMr as utiliza, tendo sido encontrada uma importante correlação entre o uso das redes sociais e a sua influência na escolha do destino, e, comparando os resultados do grupo PcDMr com o de pessoas sem deficiência (PsD), verificou-se uma correlação menor destas. Por fim, verificou-se a influência do E-WOM na escolha do destino turístico. 
Contribuições teóricas/metodológicas: apresenta os constructos e variáveis do turismo acessível e do $e$-WOM e a utilização da internet pelo turista PcDMr e como ele afeta a escolha do destino turístico por PcDMr.

Contribuições sociais / para gestão: Possibilita aos analistas de mercado, profissionais de marketing, governo, empresários e pesquisadores da área do turismo, o desenvolvimento de ações eficazes para o atendimento do turista PcDMr.

Palavras-chaves: $e$-WOM. Destino turístico. Acessibilidade. Pessoa com deficiência.

\title{
Cite como
}

American Psychological Association (APA)

Ferst, M. da C., Anjos, S. J. G. dos, Coutinho, H. R. M., \& Flores. L. C. da S. (set./dez. 2020). Electronic Word of Mouth $(e-W O M)$ e escolha do destino turístico por pessoa com deficiência ou mobilidade reduzida (PcDMr). PODIUM Sport, Leisure and Tourism Review, São Paulo, 9(3), 435-461.

https://doi.org/10.5585/podium.v9i3.16015.

\section{ELECTRONIC WORD OF MOUTH (E-WOM) AND THE CHOICE OF TOURIST DESTINATION BY DISABILITIES OR REDUCED MOBILITY PERSON}

\begin{abstract}
Objective of the study: To analyze the impact of $e$-WOM on the choice of tourist destination for people with disabilities or reduced mobility (PcDMr).
\end{abstract}

Methodology / approach: This is an explanatory study; deductive and quantitative with the use of a non-probabilistic sample for convenience, with 128 people with disabilities or reduced mobility. Data analysis was performed with the aid of SPSS Statistics software version 25. Descriptive statistics methods were used for data analysis. Analysis of the T-Test and correlation between variables.

Originality / Relevance: Academic studies on the use of existing e-WOM Existing academic studies on the use of e-WOM do not address its use by people with disabilities or reduced mobility. The academic relevance is given the growth of the accessible tourism market and the need to understand better the habits of this segment.

Main results: It was observed that the majority of respondents with disabilities or reduced mobility travel accompanied and at least once a year. As for the destination's accessibility, for PcDMr tourists it has influence or can influence the choice of tourist destination. Regarding the use of social networks, most respondents PwDMr use them, having found an important correlation between the use of social networks and their influence on the choice of destination, and, comparing the results of the PcDMr group with that of people without disabilities (PsD), there was a smaller correlation of these. Finally, the influence of E-WOM in the choice of tourist destination was verified.

Theoretical / methodological contributions: presents the constructs and variables of accessible tourism and $e-W O M$ and the internet use by the tourist PcDMr, and how it affects the choice of tourist destination by PcDMr. 
Social / Management Contributions: Enables market analysts, marketing professionals, government, (business)and tourism researchers to develop effective actions to serve the tourist PcDMr businessmen and researchers in the field of tourism to develop effective actions to assist tourists PcDMr.

Keywords: $e-W O M$. Tourist destination. Accessibility. Disabled person.

\section{BOCA A BOCA ELECTRÓNICO (E-WOM) Y LA ELECCIÓN DEL DESTINO TURÍSTICO POR PERSONAS CON DISCAPACIDAD O MOVILIDAD REDUCIDA}

\section{Resumén}

Objetivo del estudio: analizar el impacto de $e-W O M$ en la elección del destino turístico por parte de personas con discapacidad o movilidad reducida (PcDMr).

Metodología / enfoque: Este es un estudio explicativo; deductivo y cuantitativo con el uso de una muestra no probabilística por conveniencia, con 128 personas con discapacidad o movilidad reducida. El análisis de los datos se realizó con la ayuda del software SPSS Statistics versión 25. Se utilizaron métodos de estadística descriptiva para el análisis de los datos. Análisis del T-Test y correlación entre variables.

Originalidad / Relevancia: Los estudios académicos existentes sobre el uso de $e$-WOM no abordan su uso por personas con discapacidad o movilidad reducida. La relevancia académica se da por el crecimiento del mercado turístico accesible y la necesidad de conocer mejor los hábitos de este segmento.

Resultados principales: Se observó que la mayoría de los encuestados con discapacidad o movilidad reducida viajan acompañados y al menos una vez al año. En cuanto a la accesibilidad del destino, para los turistas PcDMr influye o puede influir en la elección del destino turístico. En cuanto al uso de las redes sociales, la mayoría de los encuestados PwDMr las utilizan, habiendo encontrado una importante correlación entre el uso de las redes sociales y su influencia en la elección del destino, y comparando los resultados del grupo PcDMr con el de personas sin discapacidad (PsD), hubo una correlación menor de estos. Finalmente, se verificó la influencia de E-WOM en la elección del destino turístico.

Contribuciones teóricas / metodológicas: presenta los constructos y variables de turismo accesible y $e$-WOM y el uso de internet por parte del turista PcDMr, y cómo afecta la elección del destino turístico por parte de PcDMr.

Contribuciones sociales / de gestión: permite a los analistas de mercado, comercializadores, gobiernos, empresarios e investigadores de turismo desarrollar acciones efectivas para servir al PcDMr turístico.

Palabras-claves: $e$-WOM. Destino turístico. Accesibilidad. Persona discapacitada.

\section{Introdução}

O número de pessoas com acesso à internet no mundo corresponde a aproximadamente 4 bilhões de pessoas, ou seja, mais da metade da população mundial (Kemp, 2018), sendo o uso desta na busca de informações para a escolha do destino turístico cada vez mais frequente a fim 
de obter informações corretas e relevantes sobre a viagem (Inversini, Rega, \& Zyl, 2019), melhorando a experiência turística (Adeola \& Evans, 2019).

O uso de smartphones e da internet tem proporcionado uma mudança no comportamento do consumidor turista e transformado a indústria do turismo nas últimas décadas em diversos países (Adeola \& Evans, 2019; Law, Chan, \& Wang, 2018), isso porque existe uma maior facilidade na busca de informações online. Os viajantes tem acesso a uma infinidade de informações, inclusive referências de viagens criadas por outros viajantes (Amaro, Duarte, \& Henriques, 2016) que de acordo com outras pesquisas (Fotis, Buhalis, \& Rossides, 2012) são consideradas mais confiáveis do que as opiniões ou informações de profissionais.

Embora a expansão das tecnologias de informação e comunicação (TIC) por meio das mídias sociais tenham impactado cada vez mais o comportamento e a tomada de decisão dos turistas, destacam Hua, Ramayah, Ping, \& Jacky (2017, p. 266) a ausência de achados teóricos e empíricos no setor turístico relativamente aos fatores que "podem influenciar o comportamento dos consumidores no uso das mídias sociais como ferramenta para a tomada de decisão".

No turismo, diferente de outras indústrias, tem-se uma robusta característica de intangibilidade, pois o seu principal produto é o serviço, o que comparado a outros setores, o torna muito mais vulnerável e dependente das informações sobre as suas complexas e diversas atrações (Kullada \& Michelle Kurniadjie, 2020). Disso decorre a importância de se analisar o impacto das informações buscadas na internet pelo turista.

Se a informação adequada sobre o produto ou serviço turístico é essencial para o turista e a busca por ela nas redes socias é cada vez mais frequente, o que se busca analisar neste estudo é o quanto essa informação colhida nas redes sociais: denominado E-WOM (boca a boca online), pode influenciar a escolha de um destino turístico pelo específico seguimento de turistas com deficiência ou com mobilidade reduzida (PcDMr).

A partir de 2006, com a adoção da Convenção das Nações Unidas sobre os Direitos das Pessoas com Deficiência se começou a dar maior atenção à estas pessoas, que tem o pleno direito de participar da sociedade e usufruir da mesma qualidade de vida que as pessoas sem deficiência. $\mathrm{O}$ que segmentando para a atividade turística significa ter o direito de viajar e participar das atividades de cultura e lazer (Smith, Amorim, \& Soares, 2013).

Conforme os dados do último levantamento realizado pela Organização Mundial de Saúde (World Health Organization, 2012) mais de um bilhão de pessoas em todo o mundo 
possuem algum tipo de deficiência, o que equivale a cerca de $15 \%$ da população global (Domínguez Vila, Darcy, \& González, 2015; Tsatsou, 2019). Tais dados, corroborados pelo potencial crescimento da população idosa (que frequentemente apresenta dificuldades de mobilidade, visão e audição) tem culminado em diversos estudos referentes ao turismo para pessoas com deficiência ou mobilidade reduzida (chamado de turismo acessível), que tem sido apontado por vários autores como um rentável nicho de mercado (Huh \& Singh, 2007; Babaita, 2012; Williams et al., 2017; Rabontu, 2018).

A Convenção da ONU sobre os Direitos da Pessoa com Deficiência estabelece entre seus princípios fundamentais a questão da acessibilidade, isto porque se entende que a pessoa com deficiência deve realizar suas ações cotidianas com autonomia. Em outra vertente, especula-se um grande crescimento no segmento turístico, que vem sendo constantemente descrito como uma das indústrias que mais cresce no mundo (Cole \& Morgan, 2010).

Ressalte-se que um dos princípios básicos do Código Mundial de Ética no Turismo (art. $7^{\circ}$ ) é o direito de ir e vir, além do dever universal previsto na Declaração Universal dos Direitos do Homem (ONU, 1948) de promoção dos direitos humanos e da igualdade. Importante destacar que ao se garantir o direito de ir e vir das pessoas com deficiência estar-se-á atendendo a todas as pessoas, uma vez que a acessibilidade é necessidade não apenas das pessoas com deficiência indicadas pela Organização Mundial da Saúde, mas também aos idosos, crianças, gestantes, obesos e qualquer pessoa com a mobilidade reduzida, ainda que temporariamente.

Neste contexto, considerando ainda os múltiplos benefícios para o bem-estar físico e mental da pessoa proporcionado pelo lazer e o fato de que o lazer pode implicar na melhora da capacidade cognitiva e contribuir para maior autonomia da PcDMr em suas atividades (Adam, Boakye, \& Kumi-Kyereme, 2017; Duarte, Borda, Moura, \& Spezia, 2015; Figueiredo, Eusébio, \& Kastenholz, 2012; Stumbo, Wang, \& Pegg, 2011), bem como os princípios previstos na Convenção da ONU sobre os Direitos da Pessoa com Deficiência, que lhe garantem o direito ao lazer, à cultura e ao turismo, este estudo tem como objetivo geral averiguar o impacto do $e$ WOM na escolha do destino turístico pelo turista com deficiência ou mobilidade reduzida e como objetivos específicos

i - definir os constructos e variáveis do turismo acessível;

ii - analisar o papel do E-WOM no turismo e

iii - analisar o perfil do turista com deficiência ou mobilidade reduzida no uso das redes sociais. 
Entende-se que este estudo contribuirá para os analistas de mercado, profissionais de marketing, governo, empresários e pesquisadores da área do turismo no desenvolvimento de ações eficazes para o atendimento ao público objeto desta pesquisa.

\section{Turismo acessível}

Cidadania e inclusão social são temas cada vez mais em voga em nossa sociedade e nesta perspectiva o turismo para pessoas com deficiência ou mobilidade reduzida tem se demonstrado um potencial mercado mundial em desenvolvimento (Allan, 2015; Otoo \& Kim, 2018; Rabontu, 2018).

Para fins desta pesquisa optou-se adotar o conceito de deficiência previsto na Convenção Internacional da ONU sobre os direitos da pessoa com deficiência (ONU, 2019, art. 10), que considera pessoa com deficiência as pessoas que tem impedimentos de longo prazo de natureza física, mental, intelectual ou sensorial, os quais, em interação com diversas barreiras, podem obstruir sua participação plena e efetiva na sociedade em igualdades de condições com as demais pessoas.

Desta feita, para que se garanta a participação plena e efetiva da PcDMr em igualdade de condições na sociedade, é fundamental a promoção de um meio ambiente acessível, que na perspectiva contemporânea significa acessibilidade em todas as suas vertentes, não se limitando aos espaços, mobiliários, equipamentos, edificações e transportes, mas também à informação, comunicação e tecnologias, uma vez que, para que haja real acessibilidade é preciso eliminar toda e qualquer barreira, seja ela física, arquitetônica, urbanística, comunicacional, tecnológica ou atitudinal, propiciando assim, a inclusão da PcDMr (Haegele \& Hodge, 2016; Leonardi et al., 2006).

Este turismo para pessoas com deficiência ou mobilidade reduzida tem sido denominado pela literatura como turismo acessível, que é um conjunto de instalações e práticas para permitir ou melhorar a experiência turística para um amplo espectro de pessoas (Cole \& Morgan, 2010).

Outros fatores devem ser observados no turismo acessível tais como: o atendimento da mobilidade, visão, audição e dimensões cognitivas do acesso, que devem funcionar de maneira independente, com dignidade e igualdade, por intermédio da prestação de serviços, ambientes e produtos turísticos devidamente projetados para este fim (Offei, Acheampong, AppiahBrempong, Okyere, \& Owusu, 2017). 
A Resolução $n^{\circ}$ 48/1996 da ONU que estabelece as normas sobre igualdade de oportunidades para pessoas com deficiência disciplina ser dever dos Estados membros reconhecer a importância global das condições de acessibilidade para o processo de igualdade de oportunidades em todas as esferas da vida social, devendo promover políticas públicas que visem a acessibilidade física e comunicacional, garantindo-se nos artigos 10 e 11 a acessibilidade no turismo, na cultura e no esporte a fim de que as pessoas com deficiência possam usufruir destes serviços.

O turismo de pessoas com deficiência não é apenas satisfazer às necessidades das atrações turísticas típicas, mas também é muitas vezes a única oportunidade de testar a si mesmo e suas capacidades, para adquirir novas habilidades e hábitos, superar barreiras psicológicas e começar a acreditar em si mesmo. Isso, por sua vez, leva a atitudes mais abertas e ativas em seu ambiente imediato (Zbikowski, Kuzmicki, Dabrowski, \& Soroka, 2011).

Relativamente ao turismo acessível como segmento de mercado deve-se observar que estes turistas tendem a viajar acompanhados (Cole, Zhang, Wang, \& Hu, 2019; Smith et al., 2013), bem como a crescente população de idosos, que também fazem parte do público do turismo acessível em virtude de possuírem necessidades semelhantes de acessibilidade (Dávid \& Kiss, 2011).

Como os idosos compartilham muitas das barreiras de acesso enfrentadas pelas pessoas com deficiência, ao abordar essas questões a indústria turística geraria mais negócios ao atrair mais clientes, e, ao melhorar a qualidade do serviço para pessoas com deficiência, a indústria turística melhoraria a qualidade do serviço para todos (Var, Yesiltas, Yayli, \& Öztürk, 2011).

A promoção do turismo acessível influencia na experiência do turismo por pessoas com deficiência ou mobilidade reduzida, destacando Zhang, Cole, \& Park, (2016), distintos elementos de influência em seus estudos, especificamente: conveniência, a segurança, a eficiência dos serviços, o tipo de personalidade, a sensibilidade ao meio ambiente e os fatores situacionais.

Em estudo realizado sobre produtos turísticos projetados especificamente para pessoas com deficiência, restou evidenciado haver demanda por serviços de turismo acessível melhores e mais sofisticados. (Dávid \& Kiss, 2011), além da tendência em serem mais leais e desfrutarem de estadias mais longas em suas viagens (Domínguez Vila, Alén González, \& Darcy, 2018a).

O objetivo do turismo acessível é proporcionar produtos e serviços que atendam adequadamente às necessidades das pessoas com deficiência ou mobilidade reduzida, para que 
possam aproveitar suas férias e tempo livre sem perturbações (Dávid \& Kiss, 2011). As pessoas com deficiência ou mobilidade reduzida enfrentam barreiras físicas, atitudinais, comunicacionais, informacionais e tecnológicas em suas viagens turísticas (Blichfeldt \& Nicolaisen, 2011; McKercher \& Darcy, 2018), sendo o acesso à informação um dos elementos que ganha maior importância ainda no setor turístico, em virtude do uso da internet como um canal de comunicação pela indústria turística (Domínguez Vila et al., 2018a).

A ausência de meios de transportes e serviços turísticos acessíveis influenciam negativamente na experiência turística do turista com deficiência ou mobilidade reduzida (Pühretmair \& Miesenberger, 2006), sendo um grande desafio a promoção de um turismo sem barreiras (Dávid \& Kiss, 2011; Domínguez Vila et al., 2015), uma vez que os espaços não são projetados para a utilização por PcDMr (Imrie, Pinch, \& Boyle, 1996).

Em que pese o desejo de viajar, a ausência de acessibilidade tem sido apontada como um fator impeditivo à prática do turismo por pessoas com deficiência (Daniels, Rodgers, \& Wiggins, 2005; Michopoulou, Darcy, Ambrose, \& Buhalis, 2015). Desta abordagem surge a primeira hipótese desta pesquisa $\mathrm{H} 1$ : A acessibilidade do destino turístico pode ser um fator determinante na escolha do destino pela PcDMr.

Entretanto, há que se observar a importância da informação em todo o processo de viagem da PcDMr, seja como canal de comunicação ou como compartilhador de experiências (Domínguez Vila et al., 2018a), sendo também importante observar que em virtude da ainda reinante exclusão das pessoas com deficiência (PcD), tem crescido o interesse dos pesquisadores em investigar de que forma as tecnologias e serviços digitais podem influenciar na qualidade de vida para a PcD (Tsatsou, 2019).

A partir destas premissas a respeito do uso da internet e o direito à acessibilidade, passase a analisar o papel do $e-W O M$ no turismo e o seu uso pela pessoa com deficiência ou com mobilidade reduzida.

\section{E-WOM, PcDMr e Turismo: tendências}

Pesquisas a respeito da utilização de canais digitais no turismo tem sido cada vez mais frequentes em virtude da imperiosidade de se obter visibilidade online, pois o conhecimento sobre as mídias sociais tem sido considerado vital para as empresas turísticas (Styvén \& 
Wallström, 2017) tendo a tecnologia liderado uma série de transformações no mercado (Buhalis \& Sinarta, 2019).

Com o advento da Internet, a disseminação da informação é muito mais rápida e eficaz, tendo a WEB uma funcionalidade incontestável na busca de informações e recomendações, pois fornece uma ilimitada fonte de informações com custos relativamente mínimos. Tais vantagens, "abriram o caminho para que as mídias sociais se tornassem a força mais poderosa que impulsiona o planejamento de viagens e a tomada de decisões" (Amaro et al., 2016, p. 9).

Considerando a possibilidade que os viajantes possuem de postar comentários sobre a sua viagem nas redes sociais, sites e blogs especializados, surge o "boca a boca" eletrônico ( $e$ WOM), que passa a desempenhar um importante papel na experiência de viagem do turista.

Cumpre esclarecer que antes da internet, os consumidores compartilhavam suas experiências relacionadas a produtos e serviços por intermédio do boca-a-boca tradicional (WOM), que era originalmente definido como "uma forma oral de comunicação interpessoal não comercial entre conhecidos" (Lončarić, Ribarić, \& Farkaš, 2016). Este ato de comutar informações de marketing entre os consumidores, desempenha um papel essencial na mudança de atitudes e comportamentos do consumidor em relação a produtos e serviços, uma vez ser considerada mais confiável a fonte WOM do que as mensagens empresariais (Fotis et al., 2012).

Entretanto, com os avanços tecnológicos passamos a ter uma nova forma de comunicação, o chamado boca a boca eletrônico, denominado $e$-WOM (Chu \& Kim, 2011), que se configura em todo tipo de declaração feita pelos usuários na internet sobre um produto, serviço ou empresa (Lončarić et al., 2016).

Analisando os dois conceitos se verifica que no $e$-WOM, ao contrário do WOM, a troca de informações de produtos e serviços pode acontecer não apenas no círculo de pessoas conhecidas do consumidor, mas de diversas pessoas que em dado momento utilizaram ou consumiram determinado produto ou serviço e postaram suas impressões na web, ou seja, o alcance do $e-W O M$ é muito mais amplo que do boca a boca tradicional.

Se por um lado há de fato um maior alcance do $e$-WOM, além claro, da disponibilidade e mensurabilidade deste, uma vez que fica disponível por tempo indeterminado na WEB, vale destacar que enquanto a WOM tradicional provém de um remetente que é conhecido pelo receptor da informação, a natureza eletrônica do $e-W O M$ na maioria das vezes elimina a capacidade do receptor de julgar a credibilidade do remetente e de sua mensagem justamente por não conhecê-lo (Lončarić et al., 2016). 
No turismo as informações geradas pelo e-WOM proporcionam confiabilidade e credibilidade, pois o turista pode durante o planejamento definir o seu destino por intermédio da interação virtual (Fernández-Cavia, Marchiori, Haven-Tang, \& Cantoni, 2017). Sabe-se que o planejamento de uma viagem turística é complexo e envolve muitos riscos, uma vez que o viajante irá para um destino muitas vezes desconhecido, e a possibilidade de ter diversas informações por diferentes pessoas que já visitaram o referido lugar minimiza o risco embutido e contribui para uma melhor experiência turística (Amaro et al., 2016; Leung, Sun, \& Bai, 2019).

Além de fornecer diversas informações úteis para futuros viajantes os sites da Web servem como ferramentas fundamentais para promover e vender produtos e serviços turísticos, o que possibilita o alcance de milhões de clientes em potencial em todo o mundo com um baixo investimento. E do ponto de vista do consumidor é um instrumento para encontrar e acessar fontes de informações (Smith et al., 2013).

A importância do $e$-WOM tem sido destacada pela literatura, uma vez que os indivíduos podem acessar a opinião de outros usuários com facilidade pela internet, tendo diversos estudos em várias áreas demonstrado a relação entre o número de opiniões e a venda de um produto (Raguseo \& Vitari, 2017). Como no estudo realizado por Chevalier \& Mayzlin (2006) em que demonstram que o aumento na classificação de um livro impacta no aumento de venda deste.

A influência do $e-W O M$ no comportamento do consumidor tem sido demonstrada em diversas pesquisas que o consideram mais eficaz do que os meios tradicionais de publicidade e mais influente que o tradicional WOM em virtude da sua velocidade, conveniência, alcance de um para muitos e a ausência de pressão face-to-face (Lončarić et al., 2016).

Quando se trata da decisão do destino turístico $78 \%$ dos viajantes usam fontes online na decisão deste. E ainda, 86\% utilizam a internet para definir a sua hospedagem (Tripadvisor, 2016). Considerando que viajantes com deficiência ou com mobilidade reduzida possuem um conjunto de necessidades a serem observadas para o seu conforto e bem-estar durante uma viagem, "esses turistas preferem, e muitas vezes exigem, garantias de que as instalações de viagem e os destinos de viagem são capazes de atender às suas necessidades" (Smith et al., 2013).

Ademais, a natureza efêmera, anônima e em grande escala da Internet induz novas maneiras de capturar, analisar, interpretar e gerenciar a influência que um consumidor pode ter sobre outro (Reza Jalilvand \& Samiei, 2012). Todavia, há que se ponderar a credibilidade da 
fonte da informação, uma vez que esta influencia diretamente na tomada de decisão do viajante. Impende citar o estudo realizado por Doh \& Hwang (2009) aonde afirmam que os consumidores frequentemente confiam em mensagens de e-WOM com alta credibilidade. Destacam, outrossim, relativamente à credibilidade do $e$-WOM, que apenas mensagens positivas enfraquecem a credibilidade dos sites. O resultado encontrado pelos autores sugere que algumas mensagens negativas melhoram o desempenho dos sites e a credibilidade do $e$-WOM.

Outro aspecto importante no estudo da influência do comportamento do consumidor pelas redes sociais é o engajamento, que apresenta uma valência positiva no tocante aos aspectos emocionais, comportamentais e cognitivos (Almeida, Coelho, Camilo Jr, \& Godoy, 2018). O comportamento de curtir, comentar ou marcar outra pessoa no post, conhecido como boca a boca, reflete a interação do consumidor e sinaliza consciência e engajamento, havendo estudos que apontam que $92 \%$ dos consumidores confiam mais em pessoas comuns em vez do tradicional endosso de celebridades (Amaro et al., 2016).

O objetivo de avaliar o impacto do $e$-WOM na escolha de destino turístico por pessoa com deficiência ou mobilidade reduzida surge do crescente número de turistas que usam a internet no planejamento das suas viagens agregado aos estudos que demonstram a influência da acessibilidade na prática do turismo por pessoas com deficiência ou mobilidade reduzida, uma vez que a sociedade não está preparada para atender às necessidades deste público (Smith et al., 2013). Com isso, surgem as demais hipóteses da pesquisa: H2: A pessoa com deficiência ou mobilidade reduzida faz uso das redes sociais para pesquisar o destino turístico; e H3: A pessoa com deficiência ou mobilidade reduzida é influenciada pelo $e$-WOM na escolha do destino turístico.

A internet e o uso das redes sociais podem democratizar o acesso à informação para a PcDMr (Duarte, et al., 2015) e o atendimento às suas necessidades pode ser um fator não apenas de escolha do destino turístico, mas de lealdade e fidelização (Domínguez Vila, Alén González, \& Darcy, 2018b). A pesquisa aplicada objeto deste trabalho se propõe a analisar o impacto especificamente do e-WOM no consumo de produtos e serviços turísticos por pessoas com deficiência ou mobilidade reduzida.

\section{Procedimentos metodológicos}

Relativamente aos procedimentos metodológicos trata-se de um estudo de natureza explicativa (PRODANOV; FREITAS, 2013) realizado por intermédio da análise dos conceitos 
trazidos pela literatura a respeito do turismo acessível e $e$-WOM e com a aplicação e interpretação dos dados colhidos no questionário realizado.

Quanto a forma de reflexão, utilizou-se o método dedutivo, que parte do conhecimento geral (análise da utilização da internet no planejamento turístico por PcDMr) para o particular (influência do $e-W O M$ na escolha do destino turístico por PcDMr) (Fachin, 2017), destacando Gray (2012, p. 17) que a abordagem dedutiva "avança em direção à testagem de hipóteses, depois da qual se confirma, refuta ou modifica o princípio". No caso desta pesquisa realizou-se uma revisão de literatura a partir da análise de diversos artigos científicos sobre a temática.

Por fim, trata-se de uma pesquisa quantitativa (Creswell, 2010), tendo sido realizada uma pesquisa empírica numa amostra não probabilística por conveniência com um total de 429 pessoas, das quais 128 respondentes com deficiência ou mobilidade reduzida. Para fins dos resultados apresentados foram preliminarmente considerados apenas os 128 respondentes PcDMr.

Entretanto, após a análise dos dados, considerando o número de respondentes sem deficiência e com a finalidade de enriquecer a pesquisa proporcionando uma análise comparativa da influência do $e-W O M$ por pessoas com e sem deficiência, optou-se por comparar as respostas dos dois grupos, o que culminou na análise comparativa dos 429 questionários exclusivamente nas questões referentes ao uso da internet e a sua influência na escolha do destino turístico.

Os dados foram coletados entre abril e maio de 2018, utilizando-se o instrumento de coleta de dados survey monkey. Na aplicação do questionário se utilizou a amostragem bola de neve (Cooper \& Schindler, 2016; Malhotra, 2012), em que o questionário foi replicado a partir de contatos dos pesquisadores, que divulgaram a pesquisa em suas páginas do Facebook, Instagram e Linkedin.

O formulário de coleta de dados foi um questionário composto de perguntas fechadas formuladas a partir da literatura e das hipóteses definidas neste estudo: H1: A acessibilidade do destino turístico pode ser um fator determinante na escolha do destino pela PcDMr; H2: A pessoa com deficiência ou mobilidade reduzida faz uso das redes sociais para pesquisar o destino turístico; e H3: A PcDMr é influenciada pelo e-WOM na escolha do destino turístico. O Quadro 1 demonstra os constructos e as variáveis utilizadas na elaboração do instrumento de pesquisa. 
Quadro 1 - Identificação das variáveis da pesquisa de acordo com a literatura acadêmica

\begin{tabular}{|c|c|c|c|}
\hline Autor/ano & Objeto & & Variáveis \\
\hline VAR, YEŞILTAŞ, YAYLI, & & $\begin{array}{l}\text { Dependência } \\
\text { durante a viagem }\end{array}$ & $\begin{array}{l}\text { a) Viaja sozinho } \\
\text { b) Viaja acompanhado }\end{array}$ \\
\hline $\begin{array}{l}\text { ZHANG, } \\
\text { WANG, HU (2019) }\end{array}$ & Hábitos de viagem & $\begin{array}{l}\text { Número de } \\
\text { viagens }\end{array}$ & $\begin{array}{l}\text { a) } 1 \text { vez ao ano; } \\
\text { b) } 2 \text { a } 3 \text { vezes ao ano } \\
\text { c) mais de } 3 \text { vezes ao ano }\end{array}$ \\
\hline $\begin{array}{l}\text { SMITH et al (2013); } \\
\text { COLE, ZHANG, } \\
\text { WANG, HU (2019) }\end{array}$ & $\begin{array}{l}\text { Influência da } \\
\text { acessibilidade na } \\
\text { escolha do destino } \\
\text { turístico }\end{array}$ & $\begin{array}{l}\text { Pesquisa sobre a } \\
\text { acessibilidade do } \\
\text { destino; }\end{array}$ & $\begin{array}{l}\text { a) Pesquisa, mas não influencia na } \\
\text { escolha; } \\
\text { b) Pesquisa, e influência na escolha } \\
\text { c) Pesquisa e pode influenciar na } \\
\text { escolha } \\
\text { d) Não pesquisa }\end{array}$ \\
\hline \multirow{2}{*}{$\begin{array}{l}\text { REZA JALILVAND \& } \\
\text { SAMIEI (2012); } \\
\text { TSATSOU (2019) }\end{array}$} & \multirow{2}{*}{$\begin{array}{l}\text { Condutas no } e \text { - } \\
\text { WOM }\end{array}$} & $\begin{array}{l}\text { Uso das redes } \\
\text { sociais }\end{array}$ & $\begin{array}{l}\text { a) Nunca usa as redes sociais } \\
\text { b) Raramente usa as redes sociais } \\
\text { c) Quase sempre usa as redes sociais } \\
\text { d) Sempre usa as redes sociais }\end{array}$ \\
\hline & & $\begin{array}{l}\text { Influência das } \\
\text { redes sociais para } \\
\text { a escolha do } \\
\text { destino turístico }\end{array}$ & $\begin{array}{l}\text { a) Nunca leva em consideração; } \\
\text { b) Raramente leva em consideração; } \\
\text { c) Quase sempre leva em consideração; } \\
\text { d) Sempre leva em consideração }\end{array}$ \\
\hline
\end{tabular}

Fonte: Elaborado pelos autores (2019).

A análise de dados foi realizada com auxílio do software SPSS Statistics versão 25. Utilizou-se métodos de estatística descritiva para a análise dos dados de comportamento de viagem do turista com deficiência ou mobilidade reduzida. Já para a comparação dos resultados do turista PcDMr e do turista sem deficiência nas questões referentes ao uso da internet e a sua influência na escolha do destino turístico foi realizada a análise do Teste-T e correlação entre variáveis.

\section{Resultados}

Foram analisados preliminarmente 128 questionários cuja amostra foi composta por respondentes com deficiência ou com mobilidade reduzida. Posteriormente, exclusivamente nas 
questões referentes ao uso da internet e a sua influência na escolha do destino turístico foi realizada a análise dos 429 questionários para uma análise comparativa entre os resultados do turista com deficiência ou com mobilidade reduzida para o turista sem deficiência.

Relativamente aos dados demográficos da amostra, a maioria dos respondentes com deficiência ou com mobilidade reduzida são do gênero feminino, o equivalente a $71,1 \%$ frente a $28,9 \%$ do gênero masculino. Quanto à faixa etária, 33,6\% com até 30 anos de idade; 53,1\% de 31 a 50 anos e 13,3\% acima de 51 anos. No tocante à escolaridade, a maioria - 81,2\% - tem ensino superior completo ou pós-graduação, enquanto $18,8 \%$ possui o ensino médio.

\subsection{Comportamento de viagem da pessoa com deficiência ou mobilidade reduzida}

Nos gráficos a seguir, é possível verificar o comportamento de viagem da PcDMr, sendo no Gráfico 1 dados relativamente à tendência de viajar sozinho ou acompanhado e no Gráfico 2 a frequência de viagem.

Gráfico 1 - Demonstrativo de quantidades de viagens a lazer realizadas por ano por Turistas $\mathrm{PcDMr}$

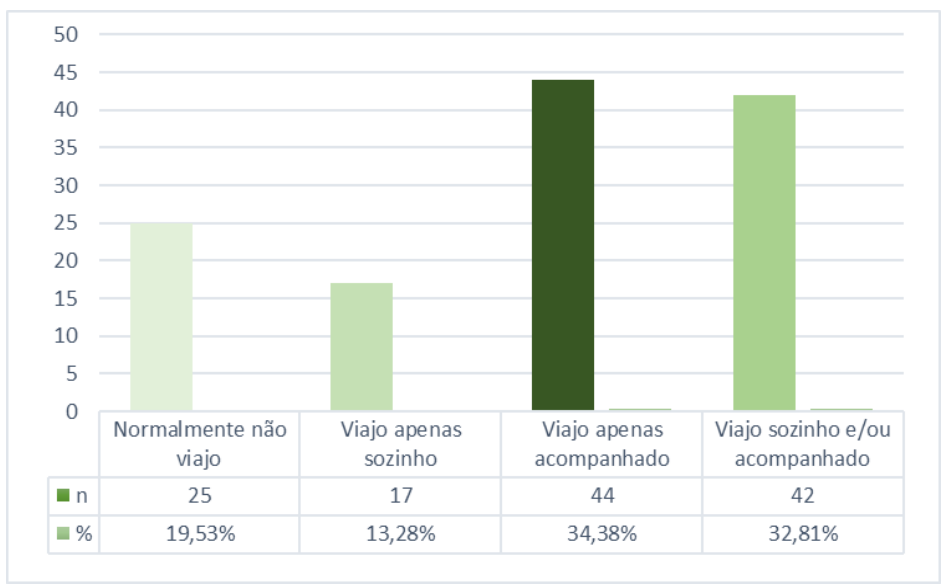

Fonte: Elaborado pelos autores (2019).

Conforme se infere do Gráfico 1, a pesquisa aplicada demonstra que dos respondentes PcDMr, 19,53\% normalmente não viaja; 34,38\% viaja apenas acompanhado; 32,81\% viaja ou sozinho ou acompanhado e somente 13,28\% viaja apenas sozinho, o que comprova os estudos anteriores da literatura que demonstram que a maioria dos viajantes com deficiência ou com mobilidade reduzida viajam acompanhados (Shu Cole et al., 2019; Var et al., 2011).

Para a análise foi questionado também (ver Gráfico 2) a respeito da frequência de viagens, e 58,59\% informou que viaja ao menos uma vez ao ano. 
Tais resultados demonstram um crescimento no número de viagens do turista PcDMr se comparados a estudos anteriores como o de Var et al (2011) que demostrou que um grande número dos viajantes PcDMr entrevistados fez mais de uma viagem nos últimos 5 anos. $\mathrm{Na}$ pesquisa de Var et al (2011) o número médio de viagens foi de 2,58. Importante destacar que no questionário aplicado nesta pesquisa se levou em consideração o número de viagens por ano, e no de Var et al (2011) as viagens nos últimos 5 (cinco) anos anteriores à pesquisa. Por outro lado, se aproxima dos estudos de Cole et al (2019) em que demostram que 77,7\% dos entrevistados haviam realizado uma viagem a lazer no último ano.

Gráfico 2 - Demonstrativo sobre a frequência de viagens por Turistas PcDMr

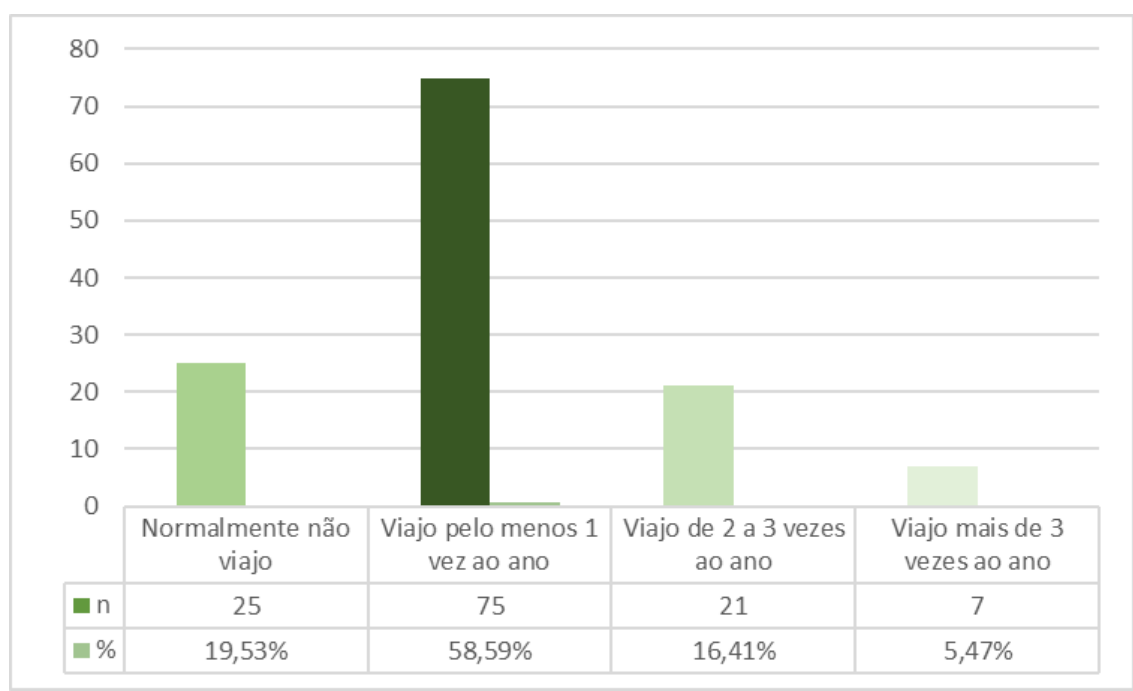

Fonte: Elaborado pelos autores (2019).

\subsection{Acessibilidade e escolha do destino turístico}

Com o objetivo de analisar o perfil do viajante $\mathrm{PcDMr}$ os respondentes foram questionados se pesquisam sobre a acessibilidade antes de decidir sobre o destino turístico e o quanto isso influencia na sua escolha. Conforme se pode inferir pela Tabela 1 somente 15,63\% dos respondentes não pesquisam sobre a acessibilidade do destino antes de decidir o seu destino turístico. Já para 66,41\% dos respondentes, a ausência de acessibilidade pode influenciar na escolha do destino turístico podendo optar por outro com melhor acessibilidade, enquanto para 17,97\% há influência da acessibilidade na escolha do destino, o que resulta na não visitação de destinos sem acessibilidade. Entretanto, $85,17 \%$ considera a acessibilidade extremamente importante, muito importante ou importante. 
Tabela 1 - Influência da acessibilidade na escolha do destino por Turistas PcDMr

\begin{tabular}{|c|c|c|}
\hline $\begin{array}{l}\text { Normalmente você pesquisa sobre acessibilidade dos destinos } \\
\text { turísticos? }\end{array}$ & $\mathbf{n}$ & $\%$ \\
\hline $\begin{array}{r}\text { Sim, e isso influencia na escolha, fazendo com que eu não visite } \\
\text { destinos sem acessibilidade }\end{array}$ & 23 & $17,97 \%$ \\
\hline $\begin{array}{r}\text { Sim, e isso pode influenciar na minha escolha, podendo optar por } \\
\text { outro que atenda melhor aos atributos de acessibilidade }\end{array}$ & 85 & $66,41 \%$ \\
\hline $\begin{array}{r}\text { Sim, mas isso não influencia na escolha, mesmo sem acessibilidade, } \\
\text { eu viajo para o destino escolhido }\end{array}$ & 0 & $0,00 \%$ \\
\hline Não & 20 & $15,63 \%$ \\
\hline $\begin{array}{l}\text { Qual o nível de importância da acessibilidade para a sua escolha } \\
\text { do destino turístico? }\end{array}$ & $\mathbf{n}$ & $\%$ \\
\hline Extremamente importante & 44 & $34,38 \%$ \\
\hline Muito importante & 29 & $22,66 \%$ \\
\hline Importante & 36 & $28,13 \%$ \\
\hline Pouco importante & 7 & $5,47 \%$ \\
\hline Não se aplica & 12 & $9,38 \%$ \\
\hline
\end{tabular}

Fonte: Elaborado pelos autores (2019).

Tais resultados demonstram que o turista PcDMr pesquisam sobre a acessibilidade do destino, bem como a sua importância para a escolha do destino turístico.

\subsection{Uso das redes sociais}

A pesquisa abordou os respondentes quanto ao seu comportamento no uso das redes sociais e a sua influência para a escolha do destino turístico (Tabela 2). 
Tabela 2 - Comportamento turista uso das redes sociais e sua influência na escolha do destino turístico por Turistas PcDMr

\begin{tabular}{|c|c|c|}
\hline Quanto ao uso das redes sociais para a escolha do destino turístico: & $\mathrm{n}$ & $\%$ \\
\hline Nunca uso as redes sociais para pesquisar sobre destinos turísticos & 12 & $9,38 \%$ \\
\hline Raramente uso as redes sociais para pesquisar sobre destinos turísticos & 32 & $25,00 \%$ \\
\hline Quase sempre uso as redes sociais para pesquisar sobre destinos turísticos & 36 & $28,13 \%$ \\
\hline Sempre uso as redes sociais para pesquisar sobre destinos turísticos & 48 & $37,50 \%$ \\
\hline $\begin{array}{l}\text { Quanto a influência das redes sociais para a escolha do destino } \\
\text { turístico: }\end{array}$ & $\mathrm{n}$ & $\%$ \\
\hline $\begin{array}{r}\text { Nunca levo em consideração os comentários nas redes sociais para escolher } \\
\text { um destino turístico }\end{array}$ & 15 & $11,72 \%$ \\
\hline $\begin{array}{r}\text { Raramente levo em consideração os comentários nas redes sociais para } \\
\text { escolher um destino turístico }\end{array}$ & 25 & $19,53 \%$ \\
\hline $\begin{array}{r}\text { Quase sempre levo em consideração os comentários nas redes sociais para } \\
\text { escolher um destino turístico }\end{array}$ & 59 & $46,09 \%$ \\
\hline $\begin{array}{r}\text { Sempre levo em consideração os comentários nas redes sociais para } \\
\text { escolher um destino turístico }\end{array}$ & 29 & $22,66 \%$ \\
\hline
\end{tabular}

Fonte: Elaborado pelos autores (2019).

Como demonstrado na Tabela 2, observou-se que 65,63\% do segmento objeto deste estudo quase sempre ou sempre utiliza as redes sociais (Facebook; Instagram; YouTube; Blogs; Booking.com; TripAdvisor, etc) para pesquisar sobre o destino turístico. Ao serem questionados sobre a influência dos comentários visualizados nas respectivas redes sociais na escolha do destino turístico 46,09\% alegou que quase sempre leva em consideração o $e$-WOM para escolher o destino turístico e $22,66 \%$ que sempre leva em consideração.

Os resultados da pesquisa estão em consonância com outros estudos que atestam a influência dos comentários nas redes sociais na aquisição de produtos e serviços turísticos (Amaro et al., 2016; Leung et al., 2019).

Ao analisar a influência dos comentários na plataforma Tripadvisor, Xie, Chen \& Wu (2015) constataram que as análises positivas online - especialmente para empresas de hotelaria e turismo - cooperam para a performance das empresas.

O diferencial desta pesquisa está em analisar a influência do $e$-WOM no específico público de pessoas com deficiência ou mobilidade reduzida, pois, em que pese os avanços nas pesquisas sobre acessibilidade, trata-se de uma área emergente na pesquisa acadêmica (Michopoulou et al., 2015) e a grande maioria das pesquisas realizadas foram dedicadas à analisar a influência de fatores ambientais, sociais e atitudinais que obstam a participação de pessoas com deficiência nas atividades turísticas (Cole et al., 2019), havendo uma carência de 
estudos que façam a triangulação do uso da tecnologia por pessoas com deficiência nas atividades turísticas.

\subsection{Análise comparativa do comportamento de pessoas com deficiência ou mobilidade reduzida e pessoas sem deficiência no uso das redes sociais para a escolha do destino}

Considerando que responderam ao questionário 429 pessoas, sendo 301 de respondentes sem deficiência ou dificuldades de mobilidade (PsD) e 128 de PcDMr, optou-se por analisar o comportamento dos dois grupos no uso das redes sociais para a escolha do destino turístico. Desta feita, foi aplicado o Teste-T para averiguar a existência ou não de similitude no comportamento dos dois grupos.

Verificou-se não haver diferenças estatísticas significantes entre os dois grupos, a exceção da pergunta sobre a importância da acessibilidade para a escolha do destino turístico, em que se encontrou uma diferença relevante da importância da acessibilidade para a pessoa com deficiência e para a pessoa sem deficiência, nos termos delimitados na Tabela 3, em que se verifica, a partir da média, que para a pessoa com deficiência ou mobilidade reduzida a acessibilidade tem maior importância do que para a pessoa sem deficiência.

Tabela 3 - Teste de amostras independentes

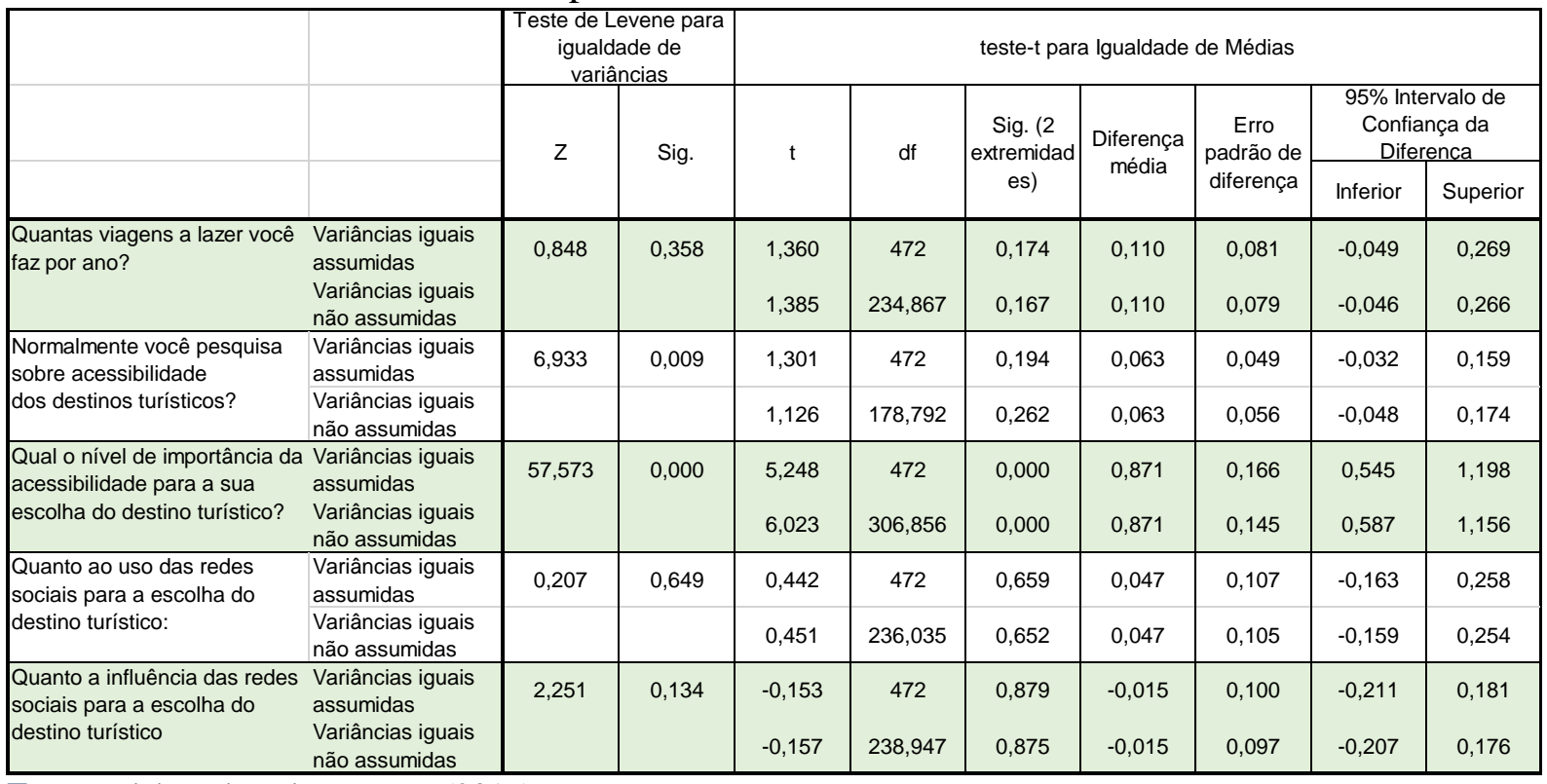

Fonte: Elaborado pelos autores (2019). 
Importante destacar que ao se analisar a correlação entre o uso das redes sociais e a sua influência para a escolha do destino turístico, encontra-se uma correlação de magnitude considerada alta pela literatura (Cohen, 1988) aplicando-se o coeficiente de correlação de Pearson, tanto no grupo de PcDMr quanto no grupo de PsD.

Entretanto, conforme se pode observar nos dados descritos na Tabela 4, a correlação entre o uso das redes sociais e a sua influência para a escolha do destino turístico é mais forte entre o grupo de PcDMr do que do grupo de PsD.

Tabela 4 - Teste de correlação de Pearson

\begin{tabular}{|c|c|c|c|c|}
\hline \multicolumn{5}{|c|}{ Correlações } \\
\hline & & & $\begin{array}{l}\text { Quanto ao uso das } \\
\text { redes sociais para a } \\
\text { escolha do destino } \\
\text { turístico: }\end{array}$ & $\begin{array}{c}\text { Quanto a influência das } \\
\text { redes sociais para a escolha } \\
\text { do destino turístico: }\end{array}$ \\
\hline \multirow{6}{*}{ PcDMr } & \multirow{3}{*}{$\begin{array}{l}\text { Quanto ao uso das } \\
\text { redes sociais para } \\
\text { a escolha do } \\
\text { destino turístico: }\end{array}$} & $\begin{array}{c}\text { Correlação de } \\
\text { Pearson }\end{array}$ & 1 &, $674^{* *}$ \\
\hline & & $\begin{array}{c}\text { Sig. (2 } \\
\text { extremidades) }\end{array}$ & & 0,000 \\
\hline & & $\mathrm{N}$ & 128 & 128 \\
\hline & \multirow{3}{*}{$\begin{array}{c}\text { Quanto a influência } \\
\text { das redes sociais } \\
\text { para a escolha do } \\
\text { destino turístico }\end{array}$} & $\begin{array}{c}\text { Correlação de } \\
\text { Pearson }\end{array}$ &, $674^{\star *}$ & 1 \\
\hline & & $\begin{array}{c}\text { Sig. (2 } \\
\text { extremidades) }\end{array}$ & 0,000 & \\
\hline & & $\mathrm{N}$ & 128 & 128 \\
\hline \multirow{6}{*}{$\begin{array}{c}\text { Pessoa } \\
\text { sem } \\
\text { deficiência }\end{array}$} & \multirow{3}{*}{$\begin{array}{l}\text { Quanto ao uso das } \\
\text { redes sociais para } \\
\text { a escolha do } \\
\text { destino turístico: }\end{array}$} & $\begin{array}{c}\text { Correlação de } \\
\text { Pearson }\end{array}$ & 1 &, $588^{\star *}$ \\
\hline & & $\begin{array}{c}\text { Sig. (2 } \\
\text { extremidades) }\end{array}$ & & 0,000 \\
\hline & & $\mathrm{N}$ & 346 & 346 \\
\hline & \multirow{3}{*}{$\begin{array}{c}\text { Quanto a influência } \\
\text { das redes sociais } \\
\text { para a escolha do } \\
\text { destino turístico }\end{array}$} & $\begin{array}{c}\text { Correlação de } \\
\text { Pearson }\end{array}$ &, $588^{* *}$ & 1 \\
\hline & & $\begin{array}{c}\text { Sig. (2 } \\
\text { extremidades) }\end{array}$ & 0,000 & \\
\hline & & $\mathrm{N}$ & 346 & 346 \\
\hline
\end{tabular}

Fonte: Elaborado pelos autores (2019).

A partir dos dados levantados se pode averiguar que há um impacto do e-WOM na escolha do destino turístico pela pessoa com deficiência ou mobilidade reduzida sendo relevante destacar que ao se analisar separadamente o grupo de PcDMr e PsD, encontrou-se uma correlação maior entre o uso das redes sociais e o impacto na escolha do destino turístico pelo turista PcDMr do que pelo turista PsD. Daí a importância de intensificar os estudos e projetos na área do turismo para o atendimento deste segmento. 
A pesquisa realizada respondeu às hipóteses levantadas, comprovando que a acessibilidade do destino turístico pode ser um fator determinante na escolha do destino para 66,41\% das PcDMr (H1) e é influente para 17,97\%; a maioria das pessoas com deficiência ou mobilidade reduzida faz uso das redes sociais para pesquisar o destino turístico (H2); $\mathrm{O} e-W O M$ influencia mais de 65\% das PcDMr na escolha do destino turístico (H3).

Vale ressaltar, ainda, o aumento da população idosa. De acordo com o Fundo de População da ONU (UNFPA), em 2015, Brasil, China, Rússia, Índia e África do Sul eram o lar de mais de 380 milhões de idosos com 60 anos ou mais, o que representava $42 \%$ da população mundial dessa faixa etária, e, considerando o ritmo de envelhecimento, os idosos dos quatro emergentes poderão somar 630 milhões em 2030 e 940 milhões em 2050 - ou 45\% do total mundial, ou seja, teremos um grupo bem considerável de possíveis viajantes idosos.

Se for considerado a pesquisa da TripAdvisor (2016), de que viajantes mais velhos tem a maior probabilidade de compartilhar comentários no TripAdvisor, é provável uma maior influência do e-WOM na escolha do destino acessível.

Desta feita, o turismo acessível não apenas é um nicho de mercado a ser considerado pela indústria do turismo conforme se verificou pela revisão de literatura (Babaita, 2012; Prochorowicz, 2012; Rabontu, 2018; Stumbo et al., 2011), como também existe uma influência considerável do $e-W O M$ na escolha de produtos e serviços turísticos por pessoa com deficiência ou mobilidade reduzida. (Lončarić et al., 2016; Niu, Wang, Yin, \& Niu, 2015; Reza Jalilvand \& Samiei, 2012; Tantawy, Kim, \& Pyo, 2005).

\section{Considerações finais}

A intangibilidade do turismo já é por si só um desafio para o viajante, uma vez que a avaliação do destino só pode ser feita a posteriori. Quando se aliam às condições de acessibilidade do destino, vem à tona mais um elemento a ser observado por profissionais do turismo e do marketing no planejamento de divulgação do destino turístico.

Ao analisar o perfil do viajante com deficiência ou com mobilidade reduzida a pesquisa realizada pelos autores comprovou as hipóteses de pesquisa levantadas. Primeiramente, no tocante a acessibilidade, para $66,41 \%$ dos respondentes a sua ausência pode influenciar na escolha do destino turístico podendo optar por outro com melhor acessibilidade, o que valida a H1 da pesquisa. Já relativamente ao uso das redes sociais pelo turista PcDMr o estudo realizado 
apontou que $65,63 \%$ do segmento objeto desta pesquisa quase sempre ou sempre utiliza as redes sociais para pesquisar sobre o destino turístico. Ao serem questionados sobre a influência dos comentários visualizados nas respectivas redes sociais na escolha do destino turístico 46,09\% alegou que quase sempre leva em consideração o $e$-WOM para escolher o destino turístico e $22,66 \%$ que sempre leva em consideração, o que comprova as hipóteses 2 e 3 apresentadas no estudo.

Demonstrou-se que a internet passou a ser um grande aliado do viajante ao permitir uma análise, ainda que por outros olhos, de todos os elementos referentes ao destino turístico, surgindo a partir desta associação da web ao setor turístico significantes desafios para o marketing de destinos (Ferreira, Ferreira, \& Paiva Neto, 2016).

E ainda que o objetivo do estudo não fosse comparar os resultados do uso das redes sociais e a influência do e-WOM para a escolha do destino turístico por turistas com e sem deficiência, é importante destacar que ao se analisar separadamente o grupo de respondentes PcDMr e PsD, encontrou-se uma correlação maior entre o uso das redes sociais e o impacto na escolha do destino turístico pelo turista $\mathrm{PcDMr}$ do que pelo turista $\mathrm{PsD}$, o que demonstra a diferença de perfil do segmento de turistas com deficiência.

Desta forma, se por um lado é fundamental para a indústria do turismo adotar conceitos básicos de turismo acessível fornecendo estruturas e serviços adaptados para garantir que esses indivíduos não sejam impedidos de viajar e encontrem suas experiências agradáveis (Smith et al., 2013), por outro lado surge para os profissionais do marketing turístico o desafio de desenvolver elementos que possibilitem a análise da acessibilidade do destino pelos viajantes.

$\mathrm{O}$ estudo corrobora outras pesquisas a respeito da influência do $e-W O M$ na escolha do serviço e do produto e sugere a aplicabilidade destas conclusões ao comportamento da pessoa com deficiência ou mobilidade reduzida. Todavia, a pesquisa apresenta algumas limitações atreladas à metodologia survey uma vez que há equívocos técnicos na construção do questionário que pode ter influenciado na compreensão da pergunta pelos respondentes. Outra limitação é a própria amostra, posto que se analisou em termos gerais o viajante com deficiência ou o viajante sem deficiência, tendo sido alcançado um baixo número de respondentes com deficiência ou mobilidade reduzida.

Sugere-se pesquisas futuras apenas com grupos de pessoas com deficiência ou mobilidade reduzida com aplicação de pesquisa de campo semiestruturada para conhecer com 
maior exatidão o perfil deste viajante e compreender o impacto da acessibilidade e do uso da internet para a atividade turística.

Cumpre salientar que turismo acessível não é apenas remoção de barreiras físicas, mas de todo tipo de barreira, ou seja, de transporte, arquitetônica, física, sensorial, comunicacional, tecnológica e atitudinal, além de proporcionar uma experiência significativa ao turista PcDMr, garantindo-lhe a igualdade de oportunidades e em plenitude, a dignidade humana.

O turismo acessível é muito mais do que um nicho de mercado. É um mandamento legal que vem sido sistematicamente ignorado pela sociedade. A influência do e-WOM neste segmento deve ser considerada e tomadas as providências necessárias para o desenvolvimento deste importante segmento turístico, fomentando a disponibilidade de informações sobre a acessibilidade do destino, pois se pode verificar a influência não apenas do $e-W O M$, mas da acessibilidade na escolha do destino turístico pelo turista PcDMr.

\section{Referências}

Adam, I., Boakye, K. A., \& Kumi-Kyereme, A. (2017). Are we our own enemies? Leisure constraints of physically and visually disabled people in Ghana. World Leisure Journal, 59(4), 272-293. https://doi.org/10.1080/16078055.2016.1245213

Adeola, O., \& Evans, O. (2019). Digital tourism: mobile phones, internet and tourism in Africa. Tourism Recreation Research, 44(2), 190/202. https://doi.org/10.1080/02508281.2018.1562662

Allan, M. (2015). Accessible tourism in Jordan: Travel constrains and motivations. European Journal of Tourism Research, 10, 109-119.

Almeida, M. I. S. de, Coelho, R. L. F., Camilo Jr, C. G., \& Godoy, R. M. F. de. (2018). Who Leads Your Opinion? Opinion Leaders' Influence on Virtual Engagement. RAC, 22(1), 115-137. https://doi.org/10.1590/1982-7849rac2018170028

Amaro, S., Duarte, P., \& Henriques, C. (2016). Travelers' use of social media: A clustering approach. Annals of Tourism Research, 59, 1-15.

https://doi.org/10.1016/j.annals.2016.03.007

Babaita, C. M. (2012). Tourism Industry in Romania and the Needs of People With Disabilities. Annals of the University of Oradea, Economic Science Series, 21(1), 481-486. Retrieved from http://search.ebscohost.com/login.aspx?direct=true \&db=buh\&AN=86068778\&site=ehostlive

Blichfeldt, B. S., \& Nicolaisen, J. (2011). Disabled travel: not easy, but doable. Current 
Ferst, M. da C., Anjos, S. J. G. dos, Coutinho, H. R. M., \& Flores. L. C. da S. (set./dez. 2020). Electronic Word of Mouth (e-WOM) e escolha do destino turístico por pessoa com deficiência ou mobilidade reduzida (PcDMr)

Issues in Tourism, 14(1), 79-102. https://doi.org/10.1080/13683500903370159

Buhalis, D., \& Sinarta, Y. (2019). Real-time co-creation and nowness service: lessons from tourism and hospitality. Journal of Travel \& Tourism Marketing, 36(5), 563-582. https://doi.org/10.1080/10548408.2019.1592059

Chevalier, J. A., \& Mayzlin, D. (2006). The effect of world of mouth on sales: Online book reviews. Journal of Marketing Research, XLIII(August), 345-354.

Chu, S.-C., \& Kim, Y. (2011). Determinants of consumer engagement in electronic word-ofmouth (eWOM) in social networking sites. International Journal of Advertising, 30(1), 4775. https://doi.org/10.2501/ija-30-1-047-075

Cohen, J. (1988). Statistical power analysis for the behavioral sciences. Hillsdale: Erlbaum.

Cole, Shu, Zhang, Y., Wang, W., \& Hu, C. (2019). The influence of accessibility and motivation on leisure travel participation of people with disabilities. Journal of Travel \& Tourism Marketing, 36(1), 119-130. https://doi.org/10.1080/10548408.2018.1496218

Cole, Stroma, \& Morgan, N. (2010). Tourism and Inequality: Problems and Prospects. London: CABI. Retrieved from https://books.google.com.br/books/about/Tourism_and_Inequality.html?id=ePAckpUDP8I C\&redir_esc=y

Cooper, D. R., \& Schindler, P. (2016). Métodos de pesquisa em administração (10a ed.). Porto Alegre: AMGH.

Creswell, J. W. (2010). Projeto de pesquisa [recurso eletrônico]: métodos qualitativo, quantitativo e misto. Tradução Magda Lopes (3a. ed.). Porto Alegre: Artmed.

Daniels, M. J., Rodgers, E. B. D., \& Wiggins, B. P. (2005). “" Travel Tales "”: an interpretive analysis of constraints and negotiations to pleasure travel as experienced by persons with physical disabilities. Tourism Management, 26, 919-930.

https://doi.org/10.1016/j.tourman.2004.06.010

Dávid, L., \& Kiss, N. (2011). Destination development and management for disabled people. Journal of Tourism Challenges \& Trends, 4(2), 113-122.

Doh, S.-J., \& Hwang, J.-S. (2009). How Consumers Evaluate eWOM (Electronic Word-ofMouth) Messages. CyberPsychology \& Behavior, 12(2), 193-197. https://doi.org/10.1089/cpb.2008.0109

Domínguez Vila, T., Alén González, E., \& Darcy, S. (2018a). Accessible tourism online resources: a Northern European perspective. Scandinavian Journal of Hospitality and Tourism, 2250(May), 1-17. https://doi.org/10.1080/15022250.2018.1478325

Domínguez Vila, T., Alén González, E., \& Darcy, S. (2018b). Website accessibility in the tourism industry: an analysis of official national tourism organization websites around the 
world. Disability and Rehabilitation, 40(24), 2895-2906.

https://doi.org/10.1080/09638288.2017.1362709

Domínguez Vila, T., Darcy, S., \& González, E. A. (2015). Competing for the disability tourism market e A comparative exploration of the factors of accessible tourism competitiveness in Spain and Australia. Tourism Management, 47. https://doi.org/http://dx.doi.org/10.1016/j.tourman.2014.10.008

Duarte, D. C., Borda, G. Z., Moura, D. G., \& Spezia, D. S. (2015). Accessible tourism in Brazil : an exploratory study about the public policies and the process of inclusion of persons with disabilities. Rb Tur - Revista Brasileira de Pesquisa Em Turismo, 9(3), 537553. https://doi.org/http://dx.doi.org/10.7784/rbtur.v9i3.863

Fachin, O. (2017). Fundamentos de metodologia: Noções básicas em pesquisa científica (6a ediçã). São Paulo: Saraiva.

Fernández-Cavia, J., Marchiori, E., Haven-Tang, C., \& Cantoni, L. (2017). Online communication in Spanish destination marketing organizations: The view of practitioners. Journal of Vacation Marketing, 23(3), 264-273. https://doi.org/10.1177/1356766716640840

Ferreira, J. R., Ferreira, L. V. F., \& Paiva Neto, A. (2016). Analysis of the official websites of tourism destinations classified socioculturally by the report the economist inteligence unit limited. Revista Visão E Ação, 18(3), 557-583. https://doi.org/10.14210/rtva.v18n2.p557583

Figueiredo, E., Eusébio, C., \& Kastenholz, E. (2012). How Diverse are Tourists with Disabilities? A Pilot Study on Accessible Leisure Tourism Experiences in Portugal. International Journal of Tourism Research, 14(6), 531-550. https://doi.org/10.1002/jtr.1913

Fotis, J., Buhalis, D., \& Rossides, N. (2012). Social Media Use and Impact during the Holiday Travel Planning Process. In Information and Communication Technologies in Tourism 2012 (pp. 13-24). Vienna: Springer Vienna. https://doi.org/10.1007/978-3-7091-1142-0_2

Gray, D. E. (2012). Pesquisa no mundo real [recurso eletrônico]. Tradução: Roberto Cataldo Costa. Porto Alegre: Penso.

Haegele, J. A., \& Hodge, S. (2016). Disability Discourse: Overview and Critiques of the Medical and Social Models. Quest, 68(2), 193-206. https://doi.org/10.1080/00336297.2016.1143849

Hua, L. Y., Ramayah, T., Ping, T. A., \& Jacky, C. J. H. (2017). Social Media as a Tool to Help Select Tourism Destinations: The Case of Malaysia. Information Systems Management, 34(3), 265-279. https://doi.org/10.1080/10580530.2017.1330004

Huh, C., \& Singh, A. J. (2007). Families Travelling with a Disabled Member: Analysing the Potential of an Emerging Niche Market Segment. Tourism and Hospitality Research, 7(4), 
Ferst, M. da C., Anjos, S. J. G. dos, Coutinho, H. R. M., \& Flores. L. C. da S. (set./dez. 2020). Electronic Word of Mouth (e-WOM) e escolha do destino turístico por pessoa com deficiência ou mobilidade reduzida (PCDMr)

212-229. https://doi.org/10.1057/palgrave.thr.6050044

Imrie, R., Pinch, S., \& Boyle, M. (1996). Identities, citizenship and power in the cities. Urban Studies, 33(8), 1255-1261. https://doi.org/10.1080/0042098966637

Inversini, A., Rega, I., \& Zyl, I. Van. (2019). Internet representations of voluntourism fail to effectively integrate tourism and volunteering. Tourism Geographies, 0(0), 1-21. https://doi.org/10.1080/14616688.2019.1600007

Kemp, S. (2018). DIGITAL IN 2018. Hootsuite. Retrieved from https://digitalreport.wearesocial.com/download

Kullada, P., \& Michelle Kurniadjie, C. R. (2020). Examining the Influence of Digital Information Quality on Tourists' Experience. Journal of Quality Assurance in Hospitality and Tourism, OO(00), 1-27. https://doi.org/10.1080/1528008X.2020.1769522

Law, R., Chan, I. C. C., \& Wang, L. (2018). A comprehensive review of mobile technology use in hospitality and tourism. Journal of Hospitality Marketing \& Management, 27(6), 626-648. https://doi.org/10.1080/19368623.2018.1423251

Leonardi, M., Bickenbach, J., Ustun, T. B., Kostanjsek, N., Chatterji, S., \& MHADIE Consortium. (2006). The definition of disability: what is in a name? Lancet (London, England), 368(9543), 1219-1221. https://doi.org/10.1016/S0140-6736(06)69498-1

Leung, X. Y., Sun, J., \& Bai, B. (2019). Thematic framework of social media research : state of the art. Tourism Review. https://doi.org/10.1108/TR-05-2018-0058

Lončarić, D., Ribarić, I., \& Farkaš, V. (2016). The role of electronic word-of-mouth in the tourism market. Tourism and Hospitality Industry. Retrieved from https://ideas.repec.org/p/tho/iscthi/15.html

Malhotra, N. K. (2012). Pesquisa de marketing [recurso eletrônico]: Uma orientação aplicada. Tradução Leme Belon ribeiro e Monica Stefani. (6a ed.). Porto Alegre: Bookman.

McKercher, B., \& Darcy, S. (2018). Re-conceptualizing barriers to travel by people with disabilities. Tourism Management Perspectives, 26, 59-66.

https://doi.org/10.1016/j.tmp.2018.01.003

Michopoulou, E., Darcy, S., Ambrose, I., \& Buhalis, D. (2015). Accessible tourism futures: the world we dream to live in and the opportunities we hope to have. Journal of Tourism Futures, 1(3), 179-188. https://doi.org/10.1108/JTF-08-2015-0043

Niu, Y., Wang, C. L., Yin, S., \& Niu, Y. (2015). How do Destination Management Organization-Led Postings Facilitate Word-of-Mouth Communications in Online Tourist Communities? A Content Analysis of China's 5A-Class Tourist Resort Websites. Journal of Travel \& Tourism Marketing, 33(7), 929-948. https://doi.org/10.1080/10548408.2015.1075455 
Offei, L., Acheampong, E., Appiah-Brempong, E., Okyere, P., \& Owusu, I. (2017). Accessibility of tourist sites to persons with disability: the case of Cape Coast and Elmina Castles in Ghana. Journal of Accessibility and Design for All, 7(2), 127-158. https://doi.org/10.17411/jacces.v7i2.112

ONU. Declaração Universal dos Direitos Humanos. (Intergovernmental Panel on Climate Change, Ed.) (1948). Cambridge University Press. https://doi.org/10.1128/AAC.03728-14

ONU. (2019). United Nations Enable | Disability. Retrieved May 9, 2019, from https://www.un.org/development/desa/disabilities

Otoo, F. E., \& Kim, S. (Sam). (2018). Analysis of studies on the travel motivations of senior tourists from 1980 to 2017: progress and future directions. Current Issues in Tourism, 1-25. https://doi.org/10.1080/13683500.2018.1540560

Prochorowicz, M. (2012). Turystyka społeczna jako forma aktywności osób niepełnosprawnych. Research Papers of the Wroclaw University of Economics, (259), 157166. Retrieved from http://search.ebscohost.com/login.aspx?direct=true \&db=bth\&AN=84371728\&lang=ptbr\&site $=$ ehost-live

Pühretmair, F., \& Miesenberger, K. (2006). Accessible Information Space to Promote Accessible Tourism. In Miesenberger K., Klaus J., Zagler W.L., Karshmer A.I. (eds) Computers Helping People with Special Needs. ICCHP 2006. Lecture Notes in Computer Science, vol 4061. Springer, Berlin, Heidelberg (pp. 329-336). https://doi.org/https://doi.org/10.1007/11788713_49

Rabontu, C. I. (2018). the Accessibility of Persons With Disabilities in Romanian Tourism. Revista de Turism - Studii Si Cercetari in Turism, 25, 1-6. Retrieved from http://www.revistadeturism.ro/rdt/article/view/401

Raguseo, E., \& Vitari, C. (2017). The effect of brand on the impact of e-WOM on hotels' financial performance. International Journal of Electronic Commerce, 21(2), 249-269. https://doi.org/10.1080/10864415.2016.1234287

Reza Jalilvand, M., \& Samiei, N. (2012). The impact of electronic word of mouth on a tourism destination choice. Emerald, 22(5), 591-612. https://doi.org/10.1108/10662241211271563

Smith, M., Amorim, E., \& Soares, C. (2013). O turismo acessível como vantagem competitiva: implicações na imagem do destino turístico. PASOS Revista de Turismo y Patrimonio Cultural, 11(3), 105-121. https://doi.org/10.25145/j.pasos.2013.11.042

Stumbo, N. J., Wang, Y., \& Pegg, S. (2011). Issues of access: What matters to people with disabilities as they seek leisure experiences. World Leisure Journal, 53(2), 91-103. https://doi.org/10.1080/04419057.2011.580549

Styvén, M. E., \& Wallström, Å. (2017). Benefits and barriers for the use of digital channels 
Ferst, M. da C., Anjos, S. J. G. dos, Coutinho, H. R. M., \& Flores. L. C. da S. (set./dez. 2020). Electronic Word of Mouth (e-WOM) e escolha do destino turístico por pessoa com deficiência ou mobilidade reduzida (PcDMr)

among small tourism companies. Scandinavian Journal of Hospitality and Tourism. https://doi.org/10.1080/15022250.2017.1379434

Tantawy, A., Kim, W. G., \& Pyo, S. (2005). Evaluation of Hotels to Accommodate Disabled Visitors Evaluation of Hotels to Accommodate Disabled Visitors. Jornal of Quality Assurnce in Hospitality \& Tourism, 5(April), 91-101. https://doi.org/10.1300/J162v05n01

Tripadvisor. (2016). TripBarometer 2016. Retrieved from https://www.tripadvisor.com/TripAdvisorInsights/wpcontent/uploads/2018/01/TripBarometer-2016-Traveler-Trends-Motivations-GlobalFindings.pdf

Tsatsou, P. (2019). Digital inclusion of people with disabilities : a qualitative study of intradisability diversity in the digital realm. Behaviour \& Information Technology. https://doi.org/10.1080/0144929X.2019.1636136

Var, T., Yesiltas, M., Yayli, A., \& Öztürk, Y. (2011). A Study on the Travel Patterns of Physically Disabled People. Asia Pacific Journal of Tourism Research, 16(December), 549-618. https://doi.org/10.1080/10941665.2011.610143

Williams, V., Tarleton, B., Heslop, P., Porter, S., Sass, B., Blue, S., ... Mason-Angelow, V. (2017). Understanding disabling barriers: a fruitful partnership between Disability Studies and social practices? Disability and Society, 33(2), 157-174.

https://doi.org/10.1080/09687599.2017.1401527

World Health Organization. (2012). The World Report on Disability. (SEDPcD, Ed.). São Paulo: SEDPcD. https://doi.org/10.1111/j.1468-3148.2012.00693.x

Xie, K. L., Chen, C., \& Wu, S. (2015). Online consumer review factors affecting offline hotel popularity : Evidence from Tripadvisor. Journal of Travel \& Tourism Marketing, (August). https://doi.org/10.1080/10548408.2015.1050538

Zbikowski, J., Kuzmicki, M., Dabrowski, D., \& Soroka, A. (2011). Vocational activity as a determinat of participation in tourism of disabled people form the area of Eastern Poland. Acta Scientiarum Polonorum, 10(2), 121-129.

Zhang, Y., Cole, S. T., \& Park, Y. W. (2016). Factors influential to tourism experiences of people with mobility impairments. Tourism Travel and Research Association: Advancing Tourism Research Globally. Retrieved from https://scholarworks.umass.edu/ttra/2012/Visual/25. 